ppi $201502 Z U 4645$

Esta publicación cientifica en formato digital es continuidad de la revista impresa ISSN-Versión Impresa 0798-1406 / ISSN-Versión on line 2542-3185Depósito legal pp

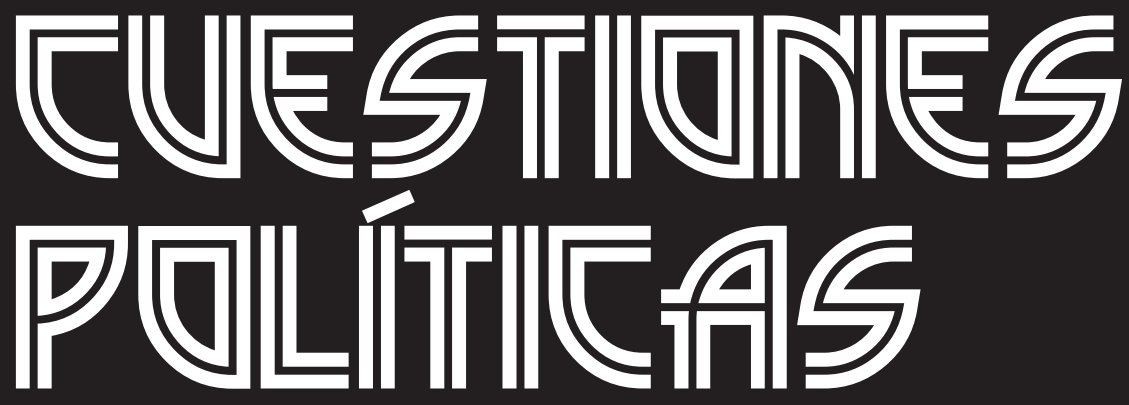

Instituto de Estudios Políticos y Derecho Público "Dr. Humberto J. La Roche' de la Facultad de Ciencias Jurídicas y Políticas de la Universidad del Zulia Maracaibo, Venezuela
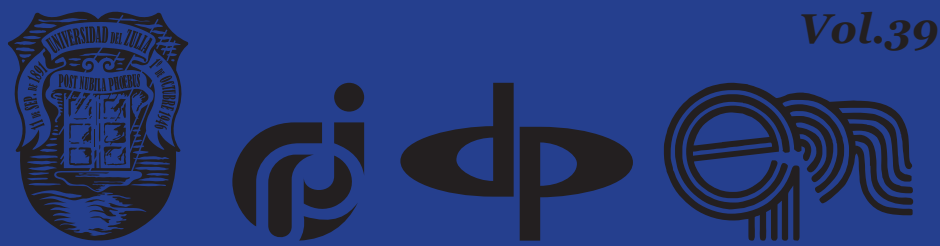


\title{
Transparencia y Gobernanza en la Gestión de la Crisis de COVID-19
}

\author{
DOI: https://doi.org/10.46398/cuestpol.3968.01
}

\section{Lorayne Finol Romero *}

\section{Resumen}

El estado de emergencia acontecido por el COVID-19 pone a prueba las capacidades de los gobiernos del mundo, en contextos de conflictividad política creciente, donde las necesidades y aspiraciones sociales de justicia y equidad crecen de forma exponencial, todo lo cual incide en la estructuración de un orden post-coronavirus con características inciertas. En este sentido, mediante una metodología documental y el análisis de datos oficiales de gestión de gobierno, el objetivo del artículo consiste en describir el sentido de las políticas de transparencia y gobernanza desplegadas en el marco de la crisis sanitaria de la pandemia del nuevo coronavirus, desde la perspectiva del gobierno abierto con énfasis especial en los casos de Chile y Colombia. Los principales hallazgos de la investigación enfatizan que la política de datos abiertos es una condición necesaria para incrementar cualitativamente los niveles de gobernanza y transparencia de la gestión pública en el manejo de la crisis, todo lo cual permite concluir que el modelo de gobierno abierto pone a disposición del ciudadano información pública que lo posiciona como vigilante de las actividades gubernamentales pero, al mismo tiempo, los datos, al ser oportunos y exhaustivos, promueven la creación de iniciativas en beneficio colectivo.

Palabras clave: transparencia en la gestión pública; gobierno abierto; crisis de COVID-19; datos abiertos; ciudadano vigilante.

Académica Investigadora adscrita a la Facultad de Derecho y Humanidades de la Universidad Central de Chile. Doctora en Derecho por la Universidad del Zulia y por la Universidad de Chile. Post doctora (c) en Ciencia Política, por la Universidad del Zulia. Ex Becaria en Gobierno Abierto e Innovación del Sector Público, en el INAP de la Universidad de Chile. ORCID ID: https://orcid.org/oooo-0oo1-70842412. Email: lorayne.finol@ucentral.cl 
 \\ Transparency and Governance in the Management of the COVID-19 Crisis
}

\begin{abstract}
The state of emergency at COVID-19 tests the capabilities of the world's governments, in contexts of increasing political conflict, where social needs and aspirations for justice and equity grow exponentially, all which impact on the structuring of a post-coronavirus order with uncertain characteristics. In this sense, through a documentary methodology and the analysis of official government management data, the objective of the article is to describe the meaning of transparency and governance policies implemented within the framework of the health crisis of the pandemic of the new coronavirus, from the perspective of the open government with special emphasis on the cases of Chile and Colombia. The main findings of the research emphasize that open data policy is a necessary condition for qualitatively increasing the levels of governance and transparency of public management in crisis management, all of which makes it possible to conclude that the open government model makes public information available to the citizen that positions him as a watchdog for government activities but, at the same time, the data, being timely and comprehensive, promote the creation of initiatives for collective benefit.
\end{abstract}

Keywords: transparency in public management; open government; COVID-19 crisis; open data; vigilant citizen.

\section{Introducción}

En los últimos años los estudios desarrollados sobre gobierno y acceso a la información pública adquieren una importancia inusitada para los científicos sociales y los agentes hacedores de las políticas públicas en general. Esto es así, por distintas y diferenciadas razones entre las que destacan al decir de Finol Romero (2018), en el mismo sentido que Criado y Ruvalcaba-Gómez (2016), la necesidad de crear un gobierno democrático acorde con los requerimientos de las sociedades de la información, que actúe en cada momento con base a los principios de rendición de cuentas, transparencia y acceso a la información de interés general. Evidentemente, estos principios fortalecen la cultura política de participación de la ciudadanía.

Recientemente, la idea de la transparencia ha cobrado aún más fuerza, considerando que las prácticas de publicación de información gubernamental, en portales web con formatos de datos abiertos son una vía importante para mejorar la calidad del gobierno, en relación proporcional al grado de su apertura (Roelofs, 2019). De lo que se trata es de construir 
un nuevo o, al menos, renovado modelo de democracia sustantiva en el siglo XXI que facilite la contraloría social de los poderes constituidos $\mathrm{y}$, especialmente, de la gestión pública en situaciones de crisis, a través del rápido acceso a la información oficial por parte de una ciudadanía informada y dispuesta a participar en la construcción de su realidad y en la realización efectiva de sus deberes y derechos cívicos, como mecanismo de alerta temprana para la disminución de contagios en medio de la pandemia.

Para los efectos partículas de esta investigación, postulamos la hipótesis de que los estado de emergencia o conmoción general de la sociedad, motivados por crisis como la sucedida en el 2020 -en el plano internacional- por el resultado devastador de la pandemia del nuevo coronavirus, son escenario propicio para apuntalar o reforzar según sea el caso, las políticas de gobierno y acceso abierto a la información como condición de posibilidad para incrementar la legitimidad del diverso abanico de políticas implementadas por los gobiernos para hacer frente a la emergencia sanitaria, políticas que más allá de sus particularidades locales y nacionales convergen en la puesta en marcha de: a) la cuarentena social, b) protocolos de bioseguridad ciudadana, c) comunicación e información sobre la prevención y tratamiento de la pandemia y, d) políticas de asistencia social a los grupos más vulnerables de la población en condición de emergencia.

En este hilo conductor, el objetivo del artículo consiste en describir el sentido de las políticas de transparencia y gobernanza en el marco de la crisis sanitaria de la pandemia del nuevo coronavirus, desde la perspectiva del gobierno abierto. Conviene clarificar que la palabra sentido se emplea para dar cuenta simplemente del propósito esencial que quieren lograr estas políticas de gestión de la crisis en su contexto particular, no obstante, no por ello se trata de una investigación propiamente dicha anclada en el método fenomenológico y hermenéutico. En lo metodológico estamos ante una investigación documentada que se sirvió del análisis, recuperación y sistematización de información impresa y digital, para la delimitación apriorística de categorías selectivas, como lo recomiendan Strauss y Corbin (2002), a partir de la revisión documental disponible en artículos científicos de alto impacto, monografías o textos académicos y cuerpos normativos, principalmente.

El trabajo en cuestión se divide en tres secciones o apartados interrelacionados, en el primero, se analiza el Impacto mundial de pandemia COVID 19, a través de un recuento de las principales consecuencias económicas, políticas y sociales que ha tenido la pandemia a nivel global, desde una visión general; en el segundo, Transparencia y Gobernanza a través de datos abiertos se describen las idea centrales que sirven para estructurar, en la teoría y en la realidad concreta, una experiencia de gobierno transparente en el manejo de la información de la gestión 
pública a través de portales data.gov; en el tercer apartado, Iniciativas de gobernanza de datos abiertos del gasto público en el contexto COVID 19, se identificaron algunas prácticas desarrolladas en distintas instancias de gobierno en Chile y Colombia para fortalecer la cultura de datos abiertos incluso en escenarios complejos de estado de emergencia. Por último, en Hallazgos y conclusiones se discuten los principales resultados de la investigación.

\section{Impacto Mundial de Pandemia COVID 19}

Cualquier balance sobre el impacto material y moral del nuevo coronavirus en el mundo actual, es cuanto menos dramático si se toma en cuenta su impronta en factores como: pérdidas de vidas humanas, huella económica global y alteración de la gobernabilidad por motivo de la crisis pandémica, tal como lo ha reseñado sistemáticamente la prensa occidental de mayor divulgación. Para el momento que se escriben estas líneas se registran más de un millón de muertes por COVID-19, siendo Asia y América del Norte las regiones del mundo más afectadas (BBC News Mundo, 2020). Incluso no se puede descartar a priori que las cepas del virus mutan rápidamente y se convierta en una enfermedad más letal, incrementando sustancialmente su índice de morbilidad.

\section{1 millón de muertos por covid-19}

\section{Datos por región}

$\square$ Asia Medio Oriente

América del Norte América Latina y el Caribe

Europa

África

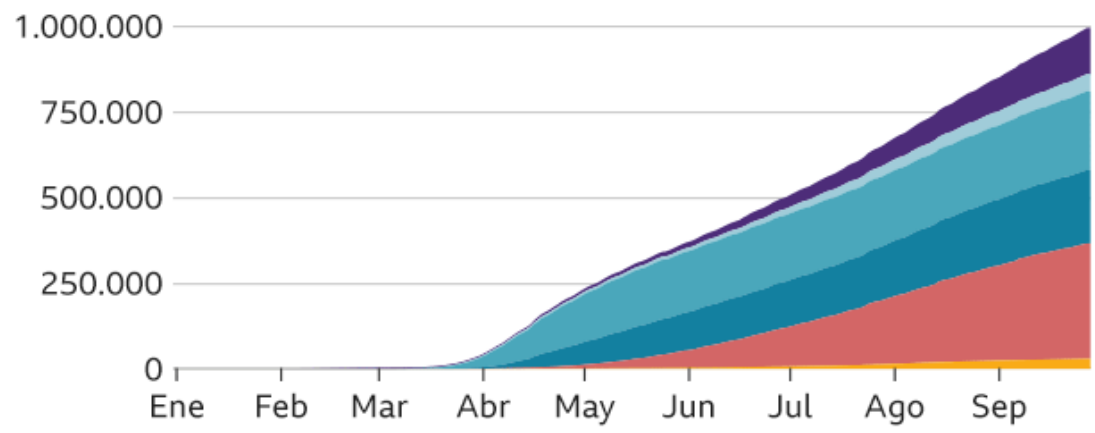

Oceanía lleva menos de 1.000 muertes registradas

Figura No. 1. Tomado de (BBC News Mundo, 2020). 
En los dominios de las finanzas públicas hay un cierto consenso en los economistas y organismos multilaterales sobre el hecho de la pandemia crea las condiciones de posibilidad para una recesión mundial, al tiempo que incrementa la pobreza neta en comunidades, países y regiones por el paro forzoso de buena parte de las actividades laborales por motivo de las políticas de confinamiento. En este sentido, según un estimado del Banco Mundial (BM), la recesión ocasionada por la pandemia se trata de la peor desde las postrimerías de la segunda guerra mundial, agravada en su decurso por la cuarentena social:

Las medidas de suspensión de las actividades que se adoptaron para contenerla han ocasionado una drástica contracción de la economía mundial, que, según las previsiones del Banco Mundial, se reducirá un 5,2 \% este año. De acuerdo con la edición de junio de 2020 del informe Perspectivas económicas mundiales del Banco, sería la peor recesión desde la Segunda Guerra Mundial, y la primera vez desde 1870 en que tantas economías experimentarían una disminución del producto per cápita (Banco Mundial, 2020: párr. 1, negritas añadidas).

En un contexto con estas características surgen preguntas como: ¿qué políticas económicas se deben implementar para gestionar la crisis y reactivar los sectores más afectados de las economías nacionales? ¿el orden posterior al COVID-19 requiere de la implementación de un nuevo o renovado modelo económico que trascienda los límites del neoliberalismo, las economías mixtas y las planificadas para generar desarrollo sostenible? ¿la nueva arquitectónica mundial implica el retorno a las economías nacionales y, a lo sumo, regionales a contravía de las tendencias globalizantes? ¿̇la recesión que está en desarrollo será un acontecimiento coyuntural o estructural? Obviamente aún no hay respuestas definitivas a las mismas y al decir de Arbeláez-Campillo y Villasmil Espinoza (2020), conviene más bien plantear de forma prospectiva algunos escenarios posibles para ilustrar los venideros contextos que dependerán en último término de las capacidades, limitaciones y recursos de cada país, así como de la eficacia y eficiencia de las políticas formuladas en cada lugar y momento.

Por su parte, la alteración de la gobernanza democrática por la pandemia no ha sido, desde nuestra percepción, lo suficientemente estudiada hasta al momento para poder determinar a ciencia cierta el verdadero impacto político del COVID-19 para los distintos actores y factores de poder. De cualquier modo, tal como refiere Arbeláez-Campillo et al. (2019), por un lado, una pandemia fuera de control puede erosionar la gobernabilidad democrática de muchas formas, bien sea al rebasar la capacidad de respuesta institucional de las instancias de gobierno dotadas de escasos recursos para hacer frente a necesidades que bien pueden aumentar de forma exponencial o; por el otro, al propagarse focos de anarquía y rebelión social motivados por los déficit en el acceso a los bienes y servicios básicos. Incluso, no debe descartarse en un contexto así una crisis humanitaria compleja en los países 
Lorayne Finol Romero
28 Transparencia y Gobernanza en la Gestión de la Crisis de COVID-19

más pobres con desplazamientos y migraciones masivas que pueden afectar a conglomerados de países.

No obstante, para los efectos concretos de las investigaciones desarrolladas en la perspectiva teórica del gobierno abierto, interesa valorar más específicamente el modo como los agentes gubernamentales y las instituciones públicas en general proporcionan información fidedigna a la ciudadanía, sobre los recursos públicos invertidos en las políticas desplegadas para gestionar la crisis y satisfacer, al mismo tiempo, las necesidades y aspiraciones sociales que este fenómeno viral ocasiona continuamente, hasta su superación definitiva. En este contexto de crisis general, formulamos la hipótesis de trabajo que postula que la transparencia no solo es una condición para la gobernanza y la gobernabilidad democrática, sino que además, es el factor clave para legitimar las políticas implementadas en los dominios de una opinión pública relativamente bien informada de su realidad, mediata e inmediata; de ahí que la combinación de herramientas de comunicación política junto el acceso abierto a la información ocupan un rol destacado en el proceso de las representaciones sociales de la pandemia.

\section{Transparencia y Gobernanza a Través de Datos Abiertos}

Recientemente la política de transparencia ha cobrado fuerza, considerando prácticas de publicación de información gubernamental proactiva, en portales web con formatos de datos abiertos de información del sector pública, bajo la premisa que estrategias de 'datos abiertos' podrían aumentar la transparencia, la participación y la eficiencia del gobierno (Sandoval-Almazan y Styrin, 2018). En este orden de ideas, su reimpulso se ha fundamentado a partir de los compromisos establecidos en los planes de acción de la Declaración de Gobierno Abierto (CEPAL, 2018) y, además, por su estrecha relación con el derecho a saber de los ciudadanos y la gobernanza en la economía digital (Bellver y Kaufman 2005; Tapscott y Agnew, 1999). Empíricamente los estudios acerca de la disponibilidad (o ausencia) de datos relevantes para las políticas, y su correlación con el tipo de régimen político, donde Hollyer et al., (2011), concluyeron que, las democracias sí son más transparentes.

A su vez, la apertura de la información pública y el gobierno abierto, concilian como un punto de encuentro, entre el fortalecimiento de la democracia y el buen gobierno (Worthy, 2015). No obstante, en América Latina sus antecedentes, se inician en la Asamblea General $75^{\circ}$ de las Naciones Unidas donde se aprobó por consenso en el año 2011, la creación de la Alianza para el Gobierno Abierto (AGA), conocida por sus siglas en inglés como 'OGP', que significan Open Government Partnership (Finol, 2018). Antes de este hito, antecedió el célebre Memorándum de Gobierno 
Abierto, del presidente de los Estados Unidos de América, Barak Obama durante su primer mandato en el año 2009 (Obama, 2009).

En este orden de ideas, también Ruvalcaba (2020) advierte que, en los últimos años, se ha presentado una tendencia en los gobiernos de diferentes niveles hacia liberar y publicar datos, no solo como un medio para generar transparencia y acceso a la información, sino también para impulsar el uso de la información en nuevas iniciativas que busquen mejorar la calidad de vida de los ciudadanos. En concordancia Birskyte (2019), señala que la transparencia en la administración pública ha tomado fuerza, convirtiéndose muchas veces en el slogan de los políticos.

Por otra parte, Rodríguez-Zepeda (2004) considera que las razones que justifican el fenómeno de la información gubernamental abierta, no es tan reciente. Pues es parte de la necesidad de fortalecer la rendición de cuentas, por su rol protagónico en la calidad de la democracia y en el proceso de incorporación de la participación ciudadana en el ejercicio del poder, así como, en la consolidación de la democracia participativa. En coherencia con Bobbio (2013), quien afirma que la democracia desde siempre ha permanecido con un halo de oscurantismo, bajo la premisa de una democracia ideal y otra real.

Sin embargo, la combinación de transparencia en portales de web de organismos del Estado con las características de los datos abiertos ha retomado un papel importante en la agenda política, a propósito del Memorándum Presidencial de Transparencia y Gobierno Abierto (Quintanilla y Gil-García, 2016). Por lo que, la revisión bibliográfica reciente refuerza la tesis que se trata de una tendencia en pleno desarrollo, según lo explican Criado et al. (2018), al señalar que, en síntesis, es una estrategia gubernamental dirigida a "abrir ventanas" en el sector público para permitir el escrutinio ciudadano a fin de reducir el oscurantismo en la información de interés público. Este mismo sentido, lo ha resumido Safarov (2019), mediante una línea de tiempo que da cuenta del proceso de incorporación del estándar de los datos abiertos, por su utilidad en múltiples dimensiones, como se puede observar en la figura 2 a continuación. 
Lorayne Finol Romero
$30 \quad$ Transparencia y Gobernanza en la Gestión de la Crisis de COVID-19

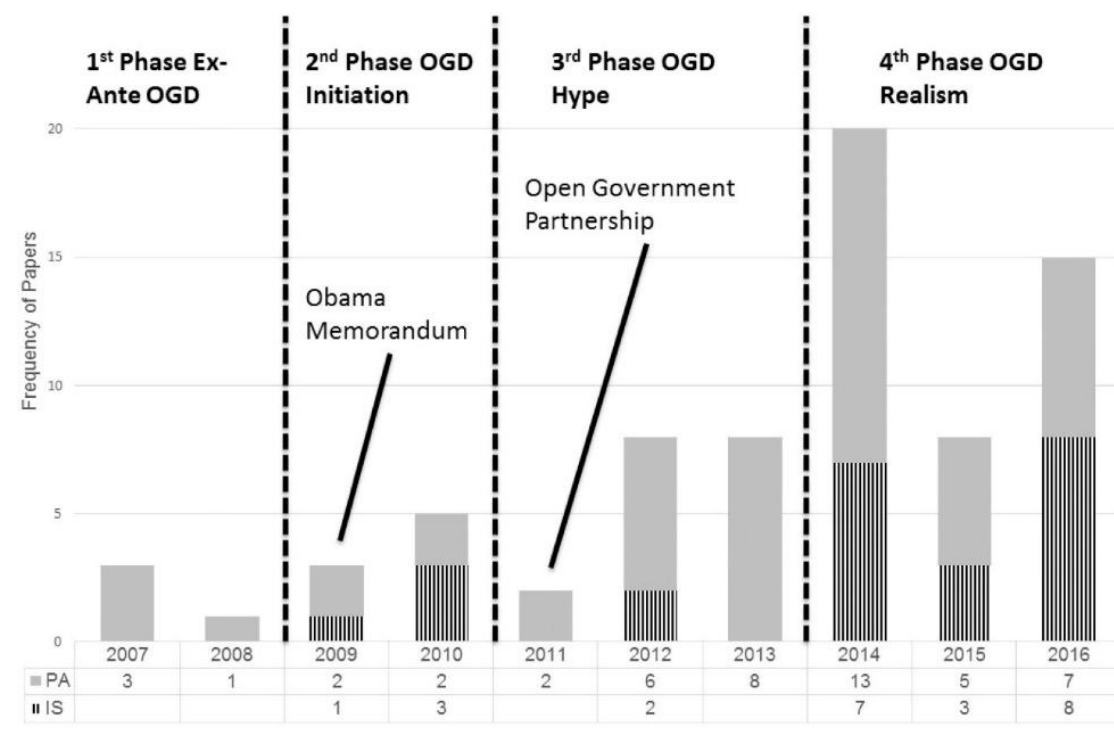

Figura No. 2. Tomado de Matheus y Janssen (2019).

Como puede observarse en la figura, los autores Matheus y Janssen (2019), en su investigación respecto a la revisión sistemática de la literatura para desentrañar la transparencia habilitado por los datos abiertos del gobierno, en inglés Open Government Data (en adelante OGD), reconocen el proceso de evolución de este fenómeno en una línea de tiempo, que inicia ex ante, la creación de la Alianza para el Gobierno Abierto en el año 2011. Esto puede ser un indicio para validar la hipótesis, que la transparencia en su dimensión dual, como la explican Meijer et al., (2018), marcó un hito importante para la institucionalización de este tipo de prácticas para la apertura del gobierno, acreditada por mejorar la transparencia y para brindar una ventana al interior del funcionamiento del gobierno, en especial referencia a que, la reutilización de la información del sector público es la consecuencia y el objeto final de la apertura de datos, donde cualquier individuo u organización aprovecha los recursos públicos expuestos por las administraciones públicas - open data gov- para cualquier propósito, incluso con fines económicos particulares por las empresas.

En este orden de ideas, el fenómeno de los datos abiertos, también se le relaciona con la llamada revolución pacífica de los datos, impulsada como un fenómeno de la cuarta revolución industrial (Ruvalcaba, 2020). Del mismo modo, como consecuencia de la incorporación de la transformación digital en la economía $\left(\mathrm{OCDE}^{2}, 2020\right)$. En forma análoga así lo consideró 
el Parlamento Europeo en la Directiva (UE) 2019/1024 (2019) que reformó a la Directiva 2003/98/CE, en donde exhorta a todos los países de la Unión a actualizar el marco legislativo conforme a los avances en las tecnologías digitales, para afrontar los obstáculos restantes y emergentes hacia la facilitación de la reutilización de la información del sector público y financiada con fondos públicos, toda ello en reconocimiento del potencial de la información del sector público para la economía y la sociedad europea.

Por otra parte, Matheus y Janssen (2019), en su estudio proponen un modelo teórico denominado The Window Theory, para la efectividad de los datos abiertos, porque existen determinantes que posibilitan o impiden la transparencia habilitada por datos gubernamentales abiertos y condicionan los efectos esperados. Todo lo anterior, es reforzado por Ruijer y Meijer (2016), quienes señalan que los datos abiertos promueven beneficios e impactos positivos en dos pilares: primero en el fortalecimiento de la confianza pública de los ciudadanos y; segundo, en el escrutinio público en escenarios sociales democráticos, cada vez más difíciles de gobernar.

En este hilo conductor, las políticas de transparencia activa a través del estándar de los datos abiertos, tiene efectos disímiles entre diferentes usuarios. Así lo explican los autores Safarov et al., (2017), cuando concluyen en su estudio que la utilización de OGD, puede tener diferentes tipos de usos y efectos, ello depende de las condiciones clave y los diferentes actores que participen en su diseño. Además, los autores antes citados, afirman que la gobernanza centralizada de OGD produce mejores resultados y un mayor nivel de implementación. En el mismo sentido, se ha pronunciado la OCDE (2020), cuando afirma que la implementación de los principios de la Carta Internacional de Datos Abiertos (IODC) evidencia en el OURdata Index, que mide el nivel de cumplimiento de los principios de la Carta entre los 33 países miembros de la OCDE y los que se encuentra en proceso de adhesión, entre ellos Colombia. Los resultados de este estudio alcanzados a través de una encuesta que tiene como objetivo medir el progreso de los países miembros, posiciona como los mejores países a Corea del Sur con 0.93 puntos, seguida de Francia con 0.90 puntos, y Colombia con 0.88.

De lo anterior se desprende que, las prácticas de publicación de la información gubernamental con características de datos abiertos, además de ser producto de los cambios suscitados con el advenimiento de la sociedad del conocimiento y la tecnología de la información y comunicación (TIC), donde el interés en la apertura de la información gubernamental y su puesta en marcha, en portales de transparencia sin restricciones legales en múltiples formatos, es lo que se conoce como Datos Abiertos (Quintanilla y Gil-García, 2016). Así se ha plasmado también en la Carta Internacional de los Datos Abiertos (2015), como datos digitales (entiéndase, información pública) son puestos a disposición de cualquier persona, en cualquier momento y en cualquier lugar, con las características técnicas y jurídicas 


\section{Lorayne Finol Romero

necesarias para que puedan ser usados, reutilizados y redistribuidos libres de costo alguno (World Web Web Fundation, 2018).

A los efectos de esta investigación, se entiende indistintamente datos o información como la razón principal que impulsa las prácticas de transparencia y gobernanza especialmente importantes, en tiempos de crisis como la pandemia COVID 19, que ha develado la desarticulación del marco legal respecto a protección de datos personales, datos abiertos y política de transparencia de la función pública, pudiendo representar un obstáculo para la efectividad de las medidas que adopten las autoridades, especialmente las sanitarias en medio de pandemias. Por ello, el esfuerzo indagatorio se concentra en describir la política de transparencia activa, datos abiertos gubernamentales, ante la inminente necesidad impostergable de conciliar acciones políticas que permitan disminuir los contagios y evitar pérdidas de vidas humanas.

Considerando que gran cantidad de los datos e información que los gobiernos recolectan de sus ciudadanos, están protegidos por la excepción al principio de máxima divulgación de la información pública, por afectar derechos fundamentales de las personas, se origina una tensión entre la obligación de transparencia activa y el derecho fundamental a la protección de datos personales (Finol, 2020). Sin perjuicio de su reconocimiento expreso en el cuarto plan nacional de acción de gobierno abierto comprendido dentro del periodo 2018-2020 del Ministerio de la Secretaría de la Presidencia de Chile (2018), en el caso de Chile, el documento contiene compromisos específicos, medibles, alcanzables, relevantes y con plazos definidos. Según los principios de la Alianza, los países parte de se comprometen a garantizar el cumplimiento de cada uno de los compromisos del Plan de Acción y deberán asegurar en forma clara cada uno de los valores de gobierno abierto a través de un proceso multisectorial y con la participación activa de la sociedad civil y la academia (Naser y Concha, 2012).

Sin perjuicio de los compromisos pactados, este esquema dicotómico se agudizó en medio de la pandemia, con un marco regulatorio desactualizado, que data de 1999 con la ley 19.628, y la ley 20.285 de 2008, además de una Norma Técnica de Datos Abiertos vigente desde el años 2013 (en proceso de reforma desde el año 2019), que pretende quitar del sustrato de la transparencia activa, prácticas de datos abiertos, dejando al margen una capacidad instalada por el Consejo para la Transparencia creado desde los inicios de la ley en el año 2009. Estas disonancias, son el común denominador en sociedades donde la transparencia es normalmente débil y se junta con altos niveles de pobreza, proyectos innecesarios, corrupción y servicios ineficientes, factores que al final, obstaculizan los esfuerzos para mejorar el gobierno, haciendo nugatorios los efectos positivos de la política en la praxis. 
La desarticulación normativa antes señalada podría ocasionar niveles inadecuados de resguardo de información personal, de igual forma, que asimetrías de información entre los diferentes estamentos públicos, impactando negativamente en las decisiones de las autoridades en materia sanitaria, haciendo referencia a los datos abiertos gubernamentales, constituidos por toda aquella información que el gobierno y sus órganos producen, gestionan, archivan y distribuyen mediante unas características específicas de una licencia abierta denominada Creative Commons (Berners-Lee, 2009).

En ese sentido se considera que, la información gubernamental publicada conforme a los principios de gobierno y datos abiertos, favorecen los procesos de toma de decisiones, impulsando una gestión gubernamental y administrativa más transparente $\mathrm{y}$, al mismo tiempo, participativa que puede promover desarrollo político, económico y sociocultural (BernersLee, 2009; OGP, 2011). Los antecedentes teóricos señalados, han dado lugar a dos instrumentos del derecho internacional, que significan un hito en esta diatriba. El primero, es la declaración de la Carta Internacional de los Datos Abiertos, de octubre de 2015 y; el segundo, es la Carta Iberoamericana para el Gobierno Abierto, en julio de 2016. Todo ello, con la finalidad de promover un consenso global sobre las características técnicas y jurídicas necesarias para garantizar la utilización, reutilización y distribución libre de la información pública en una etapa de la historia reciente, denominada la revolución mundial de los datos (Naser y Rosales, 2016).

En este contexto, la migración de la digitalización de la información gubernamental hacia formatos de datos abiertos ocupa un lugar importante en la sociedad del conocimiento y la información (Castells, 2015). Puesto que, antes del siglo XIX los estudios científicos de los asuntos públicos se centraban principalmente en la democracia formal, es decir, en la legitimidad política del gobierno, relacionada con la estructura y el funcionamiento del régimen político, verbigracia; el sistema electoral y de partidos, composición de los poderes públicos, normas de regulación, relaciones entre los niveles de gobierno, entre otros, tópicos, conocidos doctrinalmente como la democracia del sufragio (Diamond, 2003).

\section{Iniciativas de Transparencia, Gobierno y Datos Abiertos en el Contexto COVID 19}

El proceso de institucionalización de la transparencia a través del estándar de los datos abiertos evidenciado en América Latina se caracteriza por el énfasis de los gobiernos nacionales de incorporar cambios transformacionales, propios de la cuarta revolución industrial y la digitalización de procesos que afectan la vida de todos los ciudadanos (Scrollini, 2017). En particular, porque los datos abiertos permiten contar 


\section{Lorayne Finol Romero \\ 34 Transparencia y Gobernanza en la Gestión de la Crisis de COVID-19}

con nuevos mecanismos para acceder y adquirir el conocimiento, es decir, crear valor, al valerse de la información que los órganos del Estado producen en el cumplimiento de su labor pública reforzados para la apertura de la información pública proactiva, como mecanismo fundamental (Forero de Moreno, 2009; Criado et al., 2018). Lo anterior, en parte impulsado por la necesidad de hacer más transparente la rendición de cuentas a la ciudadanía, así como, de incorporar los resultados de las políticas públicas como prácticas que mejoran la gestión pública, para que pueda ser reutilizada, reproducida y difundida (Directiva 2019/1425/EU; FloresCrespo, 2013; OGP, 2011; Declaración internacional de los Datos Abiertos, 2015; Open Government Data Act, 2019).

En concordancia con la hipótesis que OGD contribuyen con la formulación de políticas basadas en evidencia en las instituciones (Safarov, 2019). Preliminarmente en esta sección antes de abordar las prácticas OGD en el marco de la pandemia por COVID-19, se analiza el estado de avance de este tipo de prácticas ex ante de la pandemia, cuya finalidad se centra en la publicación de información provista por los órganos públicos, dirigida a asegurar a las personas el efectivo ejercicio del derecho fundamental de acceso a la información pública, asimismo, como el deber de los órganos públicos de entregarla proactivamente.

Para ello, a través de un análisis descriptivo a partir de las muestras conformadas por los portales de datos abiertos centralizados de cada uno de los países latinoamericanos integrantes de la Alianza para el Gobierno Abierto, se ha retratado transversalmente la evolución de este proceso, que da cuenta de la promulgación de un conjunto de leyes y políticas en temas de transparencia y de datos abiertos, del mismo modo, que la creación de un organismo centralizado con funciones y atribuciones para la fiscalización y garantía de los estándares mínimos que coadyuven con su doble finalidad, política y administrativa. Bajo la premisa que los archivos oficiales son una gigantesca fuente de información y albergan la evidencia de las actividades que se realizaron y las que no se realizaron, de allí, radica la importancia de organizar sistemáticamente en un portal web, dado que tener un archivo desorganizado es casi lo mismo que no tenerlo, pues, aunque los datos de interés estén allí, encontrarlos será casi imposible (Torres, 2014).

La evolución de este tipo de prácticas ha quedado retratada a modo de panorama global de los datos abiertos en la región en la Tabla No 1, a continuación. 


\begin{tabular}{|c|c|c|}
\hline País & Portal OGD & Autoridad Responsable \\
\hline Argentina & $\begin{array}{l}\text { Datos Argentina Datos.Gob } \\
\text { Argentina https://datos.gob. } \\
\text { ar/ }\end{array}$ & $\begin{array}{l}\text { Secretaria de } \\
\text { Modernización Presidencia } \\
\text { de la República } \\
\end{array}$ \\
\hline Brasil & $\begin{array}{l}\text { Portal Brasileño de Datos } \\
\text { Abiertos http://dados.gov. } \\
\text { br/ }\end{array}$ & $\begin{array}{l}\text { Secretaria de Tecnología de } \\
\text { Información, Ministerio de } \\
\text { Planeamiento Desarrollo y } \\
\text { Gestión }\end{array}$ \\
\hline Chile & $\begin{array}{l}\text { Datos del gobierno abiertos } \\
\text { y transparentes https:// } \\
\text { es.datachile.io/ }\end{array}$ & \begin{tabular}{|l|} 
Ministerio Secretaría \\
General de la Presidencia \\
de Chile (SEGPRES) \\
\end{tabular} \\
\hline Colombia & $\begin{array}{l}\text { Datos Abiertos de Colombia } \\
\text { https://www.datos.gov.co/ }\end{array}$ & \begin{tabular}{|l} 
Ministerio de Tecnologías \\
de la Información y las \\
Comunicación (MINTIC) \\
\end{tabular} \\
\hline Costa Rica & $\begin{array}{l}\text { Data.gob Costa Rica } \\
\text { http://datosabiertos. } \\
\text { presidencia.go.cr }\end{array}$ & $\begin{array}{l}\text { Gobierno Abierto } \\
\text { Presidencia }\end{array}$ \\
\hline Ecuador & $\begin{array}{l}\text { Plataforma de datos abiertos } \\
\text { de Ecuador http://www. } \\
\text { datosabiertos.gob.ec/ }\end{array}$ & $\begin{array}{l}\text { Gobierno Nacional de la } \\
\text { República Administración } \\
\text { Pública Central, (APCID) } \\
\end{array}$ \\
\hline El Salvador & $\begin{array}{l}\text { Portal de Datos Abiertos El } \\
\text { Salvador https://datos.gob. } \\
\text { sv/ }\end{array}$ & $\begin{array}{l}\text { Secretaría Técnica y } \\
\text { de Planificación de } \\
\text { Presidencia Gobierno } \\
\text { Digital }\end{array}$ \\
\hline Guatemala & $\begin{array}{l}\text { Portal de Datos Abiertos } \\
\text { Guatemala https://datos. } \\
\text { minfin.gob.gt/ }\end{array}$ & $\begin{array}{l}\text { Secretaría Nacional de } \\
\text { Ciencia y Tecnología, } \\
\text {-SENACYT- } \\
\end{array}$ \\
\hline Honduras & No tiene & No tiene. \\
\hline México & $\begin{array}{l}\text { Datos Abiertos de tu } \\
\text { gobierno de México https:// } \\
\text { datos.gob.mx/ }\end{array}$ & $\begin{array}{l}\text { Secretaria de la Función } \\
\text { Pública }\end{array}$ \\
\hline Panamá & $\begin{array}{l}\text { Datos Abiertos Panamá } \\
\text { (DAP) http://www. } \\
\text { datosabiertos.gob.pa/ } \\
\end{array}$ & $\begin{array}{l}\text { Autoridad Nacional de } \\
\text { Transparencia y Acceso a } \\
\text { la Información (ANTAI) }\end{array}$ \\
\hline Paraguay & $\begin{array}{l}\text { Portal de Data.gob del } \\
\text { Paraguay https://www. } \\
\text { datos.gov.py/ }\end{array}$ & $\begin{array}{l}\text { Ministerio de Tecnología, } \\
\text { Información y } \\
\text { Comunicación (TIC) }\end{array}$ \\
\hline
\end{tabular}




\begin{tabular}{|l|l|l|}
\hline Perú & $\begin{array}{l}\text { Portal Nacional Data. } \\
\text { gob Perú https://www. } \\
\text { datosabiertos.gob.pe/ }\end{array}$ & $\begin{array}{l}\text { Secretaria del Gobierno } \\
\text { Digital Presidencia del } \\
\text { Consejo de Ministros }\end{array}$ \\
\hline Uruguay & $\begin{array}{l}\text { Catálogo Data.gob Uruguay } \\
\text { https://catalogodatos.gub. } \\
\text { uy/ }\end{array}$ & $\begin{array}{l}\text { Agencia de Gobierno } \\
\text { Electrónico y Sociedad } \\
\text { de la Información y el } \\
\text { Conocimiento de Uruguay } \\
\text { (AGESIC) }\end{array}$ \\
\hline Venezuela & No tiene & \\
\hline
\end{tabular}

Fuente: Elaboración propia.

Nota: Se incluyó Honduras pese que, a la fecha de la realización de la investigación, no cuenta con un portal de datos abiertos data.gov, no obstante, su gobierno lo ha prometido a futuro, tras su adhesión a la Alianza. No se consideran las Islas del Caribe para este estudio comparado. Tampoco Belice y Guyana, donde los dat.gov han sido impulsados por iniciativas privadas y no como una política pública.

Siguiendo con el esquema de análisis basado en categoría delimitables apriorísticamente para facilitar su comparación basada en la codificación selectiva, en el caso concreto de los portales de datos abiertos (data.gob o data.gov) en América Latina, se delimitaron tres categorías de análisis (Strauss y Corbin, 2002). La primera referida al marco institucional; la segunda, a organismos responsables de la política OGD y; la tercera, remite a su implementación como un compromiso de la Alianza. En cuanto al marco institucional se observó que la mayor parte de los países, representado por el 50\% de la muestra analizada, equivalente a 7 países de la región, han impulsado esta iniciativa como una política nacional, al alero de la modernización del Estado. Esta circunstancia, según lo explica Safarov (2019), responde a planes de gobierno con un marcado carácter presidencial y, por consiguiente, la continuidad de este tipo de iniciativas resulta amenazado producto de la poca institucionalidad política de la región. Este es el caso de Argentina, Chile, Costa Rica, Ecuador, Salvador, México y Perú, donde la gestión del portal data.gov ha sido encomendada a secretarías ministeriales de la presidencia.

En lo que concierne al segundo criterio de comparación, relativo a la organización administrativa responsable de este tipo de portales centralizados, en los países examinados solamente Panamá y Uruguay, han delegado esta función al órgano responsable de la fiscalización de las obligaciones de transparencia y derecho de acceso a la información pública, a través de Autoridad Nacional de Transparencia y Acceso a la Información, conocido por las siglas ANTAI en Panamá y a la Agencia de Gobierno Electrónico y Sociedad de la Información y el Conocimiento de Uruguay conocida por las siglas AGESIC, en Uruguay. 
Por otra parte, destacan los países que han impulsado esta iniciativa al alero del Ministerio de Ciencia, lo que puede interpretarse que su fundamentación está aparejada al fenómeno de la cuarta revolución industrial, más cercano a la revolución de los datos, que como una política presidencial propiamente dicha. Este es el caso de Paraguay, Guatemala, Colombia y Brasil. No obstante, en sentido contrario se observó en el resto de los países analizados, que el órgano responsable de la gestión de este tipo de información en el estándar dato abierto se atribuye a una secretaria o Despacho del Gobierno Digital, adscrito a la Presidencia de la República, marcando una de las características del presidencialismo acentuado de las democracias latinoamericanas (Gargarella, 2015).

En una visión de análisis comparado, respecto al tercer criterio de contrastación de los portales OGD revisados, se puede decir, que prime facie representan la puerta de entrada de la implementación de la política de apertura del gobierno con herramientas de tecnología social de última generación, sustentadas en el principio de publicidad de la información proactiva del gobierno, a la par de los avances de la era digital, en virtud, de sus múltiples beneficios de control democrático y mejoramiento de la gestión pública, que al decir de Castellano-Claramunt (2019), contribuyendo en paralelo con el debate relativo al impacto político entre la participación ciudadana e internet, donde cada país de la región ha incorporado al alero de políticas de modernización del Estado.

Sin perjuicio de aquello, hay que decir además que este tipo de iniciativas, representan el inicio del proceso OGD en América Latina, el cual puede retrotraerse al 2009, cuando dos países pioneros en este ámbito, al decir de Quintanilla y Gil-García (2016) Estados Unidos inauguró el portal data.gov y, el Reino Unido data.gov.uk, considerados por la doctrina especializada como las primeras prácticas de transparencia y apertura de información gubernamental con el estándar dato abierto, mediante portales gestionados centralizadamente a nivel de gobierno nacional y fiscalizados por un organismo responsable de la administración pública del Estado.

Por otra parte, estas prácticas ODG se observaron en los portales de Costa Rica, México, Chile, Perú, Uruguay, bajo el amparo de los compromisos del gobierno abierto. En sentido contrario, el resto de los países como, Argentina, Colombia, El Salvador Guatemala, Panamá, Paraguay, quienes se limitan a publicar la información pública, basado en el determinismo tecnológico del gobierno digital en el marco de las agendas políticas para la modernización del Estado, olvidando el principio de colaboración ciudadana, basadas en la participación de los ciudadanos en la gestión de asuntos públicos bajo la filosofía del gobierno abierto (Santa et al., 2019; Worthy, 2015).

Por lo que se refiere, a las implicancias de incorporar este tipo de iniciativas políticas como control democrático de la función pública 
impulsadas, al decir de Arenas (2016) como consecuencia del debilitamiento de la democracia y frente a la desconfianza de los ciudadanos en las instituciones políticas, hace que su incorporación como política de apertura de información pública, en los planes de gobierno abierto de cada uno de los países de la Alianza, sea verdaderamente criticable. Habida cuenta que, se han ido implementando en: "Contextos de crisis de legitimación democrática, sospechas de corrupción y debilidad gubernamental" (Guichot, 2012: 261), buscando hacer propaganda política y no como un objetivo central, de reforzar el carácter democrático de los Estados.

Por tanto, el desafío hacia la consolidación de la democracia de ciudadanos y ciudadanas informados y educados para la participación propuesta por Guillermo O `Donnell, no se ve disminuido, por el contrario, resulta agigantado, dando cuenta de una brecha inalterable entre la teoría y la práctica del debate que se cierne sobre la democracia sustantiva en el continente latinoamericano, matizada por una sensación de descontento de los ciudadanos en sus políticos y en las instituciones que los representan (Alianza para el Gobierno Abierto, 2011; O’Donnell, 2007).

\section{Datos Abiertos y COVID 19}

En el caso concreto de los portales de datos abiertos en el marco de la pandemia, en una muestra acotada conformada por Chile y Colombia, considerando como dimensión analítica los criterios del estudio de Abeleida et al., (2019), en concordancia con los principios de la Carta Internacional de los Datos Abiertos (2015), a saber: (1) Apertura, (2) Relevancia, (3) Accesibilidad y utilidad, (4) Comparación e interoperabilidad. Y con la finalidad de profundizar en el análisis descriptivo de los portales desde una perspectiva crítica se adicionaron dos indicadores específicos al tema COVID 19, la fotografía revelada en la tabla No 2, a continuación:

Tabla No 2. Portal de Datos abiertos y COVID 19: ColombiaChile.

\begin{tabular}{|l|l|l|l|}
\hline Dimensión & Indicador & Chile & Colombia \\
\hline \multirow{4}{*}{ Apertura } & $\begin{array}{l}\text { Ley de datos } \\
\text { abiertos }\end{array}$ & $\begin{array}{l}\text { Norma } \\
\text { Técnica } \\
2018\end{array}$ & Decreto 2573/2015 \\
\cline { 2 - 4 } & $\begin{array}{l}\text { Ley de acceso a } \\
\text { la Información }\end{array}$ & Ley 20285 & $\begin{array}{l}\text { Ley de Transparencia y } \\
\text { Derecho de Acceso a la } \\
\text { Información, No 1712. }\end{array}$ \\
\hline
\end{tabular}




\begin{tabular}{|c|c|c|c|}
\hline \multirow{2}{*}{ Relevancia } & $\begin{array}{l}\text { Política } \\
\text { modernización } \\
\text { del Estado }\end{array}$ & $\begin{array}{l}\text { Política de } \\
\text { gobierno } \\
\text { digital }\end{array}$ & $\begin{array}{l}\text { Agenda de } \\
\text { modernización del } \\
\text { Estado }\end{array}$ \\
\hline & $\begin{array}{l}\text { Compromiso del } \\
\text { Gobierno Abierto }\end{array}$ & $\begin{array}{l}\text { Instrucción } \\
\text { presidencial } \\
\mathrm{N}^{\mathrm{O}} 5 .\end{array}$ & No \\
\hline $\begin{array}{l}\text { Accesibilidad } \\
\text { y utilidad }\end{array}$ & $\begin{array}{l}\text { Barreras } \\
\text { burocráticas ni } \\
\text { administrativas } \\
\text { para la Re- } \\
\text { Utilización }\end{array}$ & $\begin{array}{l}\text { No hay que } \\
\text { registrarse }\end{array}$ & $\begin{array}{l}\text { Hay que registrarse } \\
\text { para acceder y publicar } \\
\text { los datos }\end{array}$ \\
\hline \multirow{2}{*}{$\begin{array}{l}\text { Comparación } \\
\text { e interopera- } \\
\text { bilidad }\end{array}$} & Comparables & $\begin{array}{l}\text { Información } \\
\text { publicada } \\
\text { al menos } 4 \\
\text { formatos }\end{array}$ & $\begin{array}{l}\text { Información publicada } \\
\text { al menos } 4 \text { formatos }\end{array}$ \\
\hline & Interoperables & $\begin{array}{l}2166 \\
\text { organismos }\end{array}$ & 1184 organismos \\
\hline \multirow[t]{2}{*}{$\begin{array}{l}\text { Datos } \\
\text { abiertos } \\
\text { COVID } 19\end{array}$} & $\begin{array}{l}\text { Temas } \\
\text { publicados }\end{array}$ & 8 & $\begin{array}{l}\text { Programas sociales } \\
\text { (1), Transferencias } \\
\text { interterritoriales } \\
\text { fiscales (1), Impacto } \\
\text { económico COVID en } \\
\text { el sector minería (4) } \\
\end{array}$ \\
\hline & $\begin{array}{l}\text { Cantidad de } \\
\text { conjunto de } \\
\text { datos abiertos } \\
\text { (data sets) }\end{array}$ & 127 & $\begin{array}{l}\text { (125) Salud y } \\
\text { Protección, } \\
\text { (1) Justicia y Derecho, } \\
\text { (1) Convivencia } \\
\text { durante la pandemia }\end{array}$ \\
\hline
\end{tabular}

Fuente: Elaboración propia (2020) a partir de Abeleida et al., (2019). Nota: Estas observaciones se dieron lugar a la fecha 29 de octubre de 2020, por lo tanto, las modificaciones posteriores, no pudieron ser incorporadas en este análisis.

Respecto a los datos abiertos en el marco de la pandemia, se pudo observar en el portal de Chile, la publicación proactiva de 8 conjuntos de datos abiertos, es decir, de información pública que cumple con los principios de la Carta Internacional de los Datos Abiertos. De la información disponible en el portal data.gob.cl, solo uno de ellos, da cuenta del Programa alimentos para Chile, publicado por Municipalidad de Peñalolén, en donde la municipalidad rinde cuentas del listado que contiene información respecto de la cantidad de cajas distribuidas según unidad vecinal, junto con información de mermas producidas. 


\section{0 \\ Lorayne Finol Romero \\ Transparencia y Gobernanza en la Gestión de la Crisis de COVID-19}

Por otra parte, se observó la publicación de otro conjunto de datos, relativos a Información de gastos municipales con aportes Gobierno Central, igualmente publicado por Municipalidad de Peñalolén. Esta rendición de cuenta del gasto fiscal es consecuencia de los mecanismos de financiación transparente en el medio de la emergencia sanitaria, que obligó al gobierno central a realizar aportes a los municipios para el apoyo con los gastos derivados producto de la necesidad de vecinas y vecinos. Ambos datasets, publicados en formatos abiertos editables en el portal centralizado de datos abiertos de Chile, lo que da cuenta de la verdadera utilidad de este tipo de prácticas, dirigidas a rendir cuentas y además a la reutilización por la población.

Respecto a otra información publicada en el portal data.gob Chile, en el marco de la pandemia, se concentra en el rubro minero del cobre, específicamente información relativa a los proyectos mineros afectados por COVID-19, publicado por Ministerio de Minería, en donde da cuenta de los proyectos mineros, de diversas pastas, afectados por la aparición del COVID-19, del mismo modo, se observó información relativa a las faenas de cobre afectados por COVID-19, publicado por Ministerio de Minería -y considera el impacto en la producción de las faenas operativas de cobre por la aparición del COVID-19-.

Por otra parte, también entrega información en datos abiertos, en temas relativos a las faenas de oro afectados por COVID-19, por parte del Ministerio de Minería, en donde producto de la reducción de las faenas considera el impacto en la producción por la aparición del COVID-19. Lo mismo, en el caso de las faenas de plata afectados por COVID-19, donde el Ministerio de Minería, considera el impacto en la producción de las faenas operativas de plata por la aparición del COVID-19 y, por último, el mismo, rinde cuenta de la evolución del precio del cobre por COVID-19 en Chile, desde primer caso de COVID-19 el 3 de marzo en Talca. Respecto a las características de la información publicada, cabe destacar, que los conjuntos de datos, o data sets observados, cumplen con el estándar de los datos abiertos, dado que están publicados en formatos editables, estructurados en CSV; XLS; XLSX.

En lo que respecta al portal de datos abiertos de Colombia, se pudo observar una mayor cantidad y calidad de datos abiertos publicados en el portal, en lo que se refiere al tema COVID 19, además, resaltar que la información disponible gravita en torno al área de Protección y Salud, excepto dos datasets diferenciados, relativos a Justicia y Derecho y Convivencia Ciudadana. Es importante señalar, que este análisis descriptivo pretende resignificar la información publicada, es decir, no basta con publicar grandes cantidades de información pública en estos portales, si esta información no guarda relación con un propósito mayor, que pasa por orientar la rendición de cuentas a la ciudadanía y permitirle participar en los procesos de toma de decisiones políticas, como un sistema de alerta temprana para la prevención de los contagios. 
En este aspecto crucial conviene detenerse en lo que señala Criado et al. (2018), respecto al verdadero propósito que persiguen los OGD, el cual no solo se centra en facilitar el escrutinio ciudadano a fin de reducir el oscurantismo, pues, de conformidad con los principios de la carta internacional de los datos abiertos (2015), específicamente en el Principio VI éstos también permiten impulsar el desarrollo incluyente y la innovación, según el cual, la reutilización de la información del sector público, conlleva un conjunto de externalidades positivas, tanto para fines comerciales como no comerciales, además de promover la creatividad y la innovación.

Bajo esta premisa, gobiernos, ciudadanos, instituciones académicas y organizaciones de la sociedad civil y del sector público mediante su reutilización crean valor público, con miras a propender beneficios sociales y económicos a todos los sectores (Moore, 1997; Mulgan, 2016). En el mismo orden de ideas, lo describen Margetts y Dorobantu (2019), quienes señalan que actualmente se producen más de 2.5 quintillones de bytes de datos cada día y, las empresas del sector privado ven en este fenómeno una oportunidad para impulsar modelos de inteligencia artificial que puedan procesar este alto tráfico de información, así como, modelos de negocios cuya materia prima la configura la información pública generada en estos portales de datos abiertos gubernamentales.

Bajo esta premisa, podría pensarse que una menor cantidad de información del Estado disponibilizada en estos portales, podría significar una ralentización de la activación económica post coronavirus, lo contrario, mayor disponibilización de datos abiertos en el portal data gov/gob, de conformidad con la Directiva 1425/2019(EU), sería un catalizador de la economía digital, validando la hipótesis de los estudios empíricos, como el de Shambaugh y Shen (2018), quienes demostraron que los niveles más altos de transparencia del gobierno están fuertemente relacionados con períodos más cortos de inflación y crisis monetarias. En forma análoga, lo describen Muente y Serale (2019), quienes los comparan con el oro negro de esta era.

De igual modo Mueller y Engewald (2018), quienes señalan que están ventanas de información son oportunidades de acceder a información oficial de las autoridades públicas y, por tanto, de aumentar el nivel de transparencia la efectividad de estas políticas, puede incrementar la confianza en el gobierno, además, de contribuir con el tercer pilar de la calidad de la democracia propuesta por Morlino (2007), y ratificado por Meijer et al. (2018) cuando afirman, que en una democracia de calidad los ciudadanos tienen el:

(...) poder de controlar y evaluar cómo se realizan a través del pleno respeto de las normas vigentes, el llamado rule of law, su eficiente aplicación, la eficacia en la toma de las decisiones junto a la responsabilidad política por las elecciones tomadas por el personal elegido también (Morlino, 2007: 6). 


\section{2 \\ Lorayne Finol Romero \\ Transparencia y Gobernanza en la Gestión de la Crisis de COVID-19}

En relación con el concepto de calidad de la democracia antes señalado, la información gubernamental abierta, pasa a ser un instrumento que impulsa una mejor comprensión de determinadas situaciones o problemas sociales y, así mejorar los procesos de toma de decisiones y el diseño de políticas públicas preventivas en resguardo de la salud pública (Quintanilla y Gil-Gracia, 2016). En este hilo conductor, podría disminuir la corrupción, fortalecer la democracia y la legitimidad de los gobiernos. En ese sentido, la información pública se convierte en el núcleo medular del proceso de rendición de cuentas, por tanto, su accesibilidad se tutela como el derecho humano universal de acceso a la información pública. En ese sentido, se ha conducido el desarrollo legislativo evidenciado en el derecho internacional latinoamericano en el último periodo, en coherencia con el movimiento denominado la revolución de los datos y del reconocimiento progresivo del derecho de acceso a la información pública como un derecho humano.

\section{Hallazgos y Conclusiones}

Todo indica que es muy recomendable que la publicación de información de los estamentos gubernamentales debe ser gestionada por agencias especializadas y centralizadamente, dada la amplia gama de conjuntos de datos disponibles. Este tipo de prácticas de transparencia y gobernanza a través de datos abiertos publicados en portales data.gov, guardan relación con la hipótesis que afirma que un gobierno abierto, es un modelo de gestión donde los políticos responsables del gobierno, abren ventanas de información al mundo a través de portales web, además que, inter-operan en red con los demás actores del sistema político; compartiendo información que anteriormente estaba celosamente guardada, potenciando el poder de la colaboración masiva y, la transparencia en todas sus operaciones para innovar junto con la ciudadanía, en la creación de soluciones a las demandas de una sociedad cada vez más informada y difícil de gobernar.

Es decir, además de poner a disposición del ciudadano información pública proactivamente que permite darle herramientas como un observador $\mathrm{y}$ vigilante de las actividades gubernamentales, al mismo tiempo, los datos, al ser oportunos y exhaustivos, conforme al Principio II de la Carta Internacional de los datos abiertos, promueve la creación de iniciativas en beneficio colectivo. En este sentido, hay que diferenciar la oportunidad y exhaustividad de los datos abiertos observados en el portal de Colombia en contraste con el rezago observado en el portal de Chile. Podría pensarse que esto se debe a que, para ello, se requiere de tiempo, recursos humanos y técnicos para identificar los datos que pueden ser liberados o publicados, a fin de saber qué datos priorizar para su liberación y mejora, datos que deben ser exhaustivos, precisos y de alta calidad. 
Sin perjuicio que en los años setenta, la frase de información pública del gobierno abierto se vinculó con el secreto gubernamental y de restricciones del derecho a saber de las decisiones públicas del Reino Unido (SandovalAlmazán et al., 2018), hoy en día, en sentido contrario y en convergencia con la llamada revolución de los datos, también conocida como la economía de los datos (Agenda Digital para la Unión Europea), se ha impulsado un conjunto de reformas globales, dirigidas a fortalecer la implementación de prácticas de gobernanza y datos abiertos, que procuran la máxima eficiencia de la acción pública, así como, el rescate de la confianza de los ciudadanos, en consonancia los Principios de la Alianza para el gobierno Abierto (AGA) suscrita en el año 2011.

En el mismo orden de ideas, fue reconocida como uno de los Objetivos para el Desarrollo Sostenible en la Agenda 2030, por la Asamblea General de las Naciones Unidas (ODS, 2015). De igual modo, Sandoval-Almazan y Styrin (2018); así como, Criado et al, (2018), al igual, como ya se mencionó Safarov et al. (2017), quienes, con sus estudios empíricos han reanimado el debate público contemporáneo a reimpulsar la apertura y la reutilización de la información pública, en virtud, de sus efectos en tres dimensiones.

Estas iniciativas, por un lado, hacen más participativa la acción estatal, al exigir que en la formulación de políticas se incorporen evidencias, así como, la colaboración ciudadana y las buenas prácticas aplicadas. La iniciativa de gobierno abierto, fue re-impulsada por la Ley Pública 115-435 promulgada en Estados Unidos el 14 de enero de 2019 identificada como Open Government Data Act., 2019, en donde se exige a las agencias federales que las políticas sean abiertas, es decir, que promuevan la participación ciudadana y, además, que los datos de la agencias gubernamentales estén disponibles, como insumos necesarios para la elaboración de políticas, así como su re-utilización para emprendimientos económicos y servicios públicos que generen valor social.

Por el otro, la apertura de la información pública también promueve transparencia y rendición de cuentas, núcleo central para la superación de la escasa credibilidad de los ciudadanos en las instituciones políticas y sus gobernantes, haciéndolas más transparentes, responsables y receptivas (Morlino, 2007, OCDE, 2020). Y, por último, los datos abiertos permiten a los usuarios identificar, comparar, inequidades económicas y sociales, para la toma de decisiones informadas (Lathrop y Ruma, 2010). Por su parte, los países de América Latina, a través de iniciativas legales centradas en favorecer la transparencia de la gestión pública y el acceso a la información gubernamental, han iniciado un proceso de reformas legislativas y reimpulso de prácticas de gobierno, dirigidas a promover un rediseño del rol del Estado, en coherencia con el fenómeno de la revolución de los datos.

Quedan pendiente para futuras investigaciones sobre gobierno abierto y acceso abierto a la información, valorar cualitativa y cuantitativamente la 


\section{Lorayne Finol Romero

forma como las campañas de comunicación política sobre el COVID-19 y sus consecuentes normas de bioseguridad, por ejemplo, manejan ingentes cantidades de recursos que deben ser publicadas exhaustivamente en los portales de las diversas instancias de gobiernas que las promocionan, como condición necesaria para determinar en una relación costo-beneficio, su éxito, limitaciones o fracasos según el contexto, en un ejercicio reflexivo e indagatorio que combina en igualdad de condiciones el derecho público, evaluación de las políticas públicas y la ciencia política, entre otras ciencia sociales.

\section{Referencias Bibliográficas}

ABELEIDA, Carolina; CARVAJAL GONZÁLEZ, Javiera; FINOL, Lorayne; MANZANO CHÁVEZ, Liliana. 2019. "Avances de la transparencia activa y datos abiertos en gobiernos locales: el caso de Coquimbo y La Serena en Chile" En: Revista Iberoamericana de Estudios Municipales (RIEM). Disponible en línea. En: https://doi.org/10.32457/riem.vi20.314. Fecha de consulta: 10/10/2020.

ARBELÁEZ-CAMPILLO, Diego F; ANDREYEVNA DUDAREVA, Marianna; ROJAS-BAHAMÓN, Magda Julissa. 2019. "Las pandemias como factor perturbador del orden geopolítico del mundo globalizado" En: Cuestiones Políticas. Vol. 36, No. 63, pp. 134-150.

ARBELÁEZ-CAMPILLO,DiegoF;VillasmilEspinoza,JorgeJ.2020."Escenarios prospectivos de un nuevo orden internacional que se vislumbra luego de la pandemia COVID-19" En: Telos. Disponible en línea. En: http://www. doi.org/10.36390/telos223.02. Fecha de consulta: 10/10/2020.

ARENAS RAMIRO, Mónica. 2016. “Transparencia, acceso a la información públicaydemocracia: Elementosinseparables"En: Revista Transparencia \& Sociedad. No. 4, pp. 113-131.

BANCO MUNDIAL. 2020. "Banco Mundial Birf Aif" En: La COVID-19 (coronavirus) hunde a la economía mundial en la peor recesión desde la Segunda Guerra Mundial. Disponible en línea. En: https://www. bancomundial.org/es/news. Fecha de consulta: 08/06/2020

BBC NEWS MUNDO. 2020. "BBC News Mundo. Obtenido de 1 millón de muertes por coronavirus: el gráfico que muestra cuáles son las regiones más golpeadas por la pandemia” Disponible en línea. En: https://www. bbc.com/mundo/noticias-internacional-54335416. Fecha de consulta: o8/06/2020. 
BELLVER, Ana; KAUFMANN, Daniel. 2005. "Transparenting Transparency: Initial Empirics and Policy Applications" Borrador del documento de debate presentado en la conferencia del FMI sobre transparencia e integridad del 6 al 7 de julio de 2005, Banco Mundial, Washington, DC. Disponible en línea. En: https://gsdrc.org/document-library/ transparenting-transparency-initial-empirics-and-policy-applications/. Fecha de consulta: 08/04/ 2019.

BERNERS-LEE, Tim. 2009. Linked data - Design issues. Disponible en línea. En: http://www.w3.org/DesignIssues/LinkedData.html. Fecha de consulta: 08/04/ 2019.

BIRSKYTE, Liucija. 2019. "Determinants of Budget Transparency in Lithuanian Municipalities" En: Public Performance and Management Review. Disponible en línea. En: https://doi.org/10.108o/15309576.2018.15079 15. Fecha de consulta: 08/04/ 2019.

BOBBIO, Norberto. 2013. Democracia y Secreto. Centzontle/ Fondo Cultura Económica. México DF., Mexico.

CASTELLANO-CLARAMUNT, Jorge. C. 2019. "Las imbricaciones políticas entre la participación ciudadana e Internet” En: Revista de Derecho Político. Vol. 1, No.106, pp. 167-197.

CASTELLS, Manuel. 2015. "La era de la información: economía, sociedad y cultura", Vol. 1. La sociedad red. Novena edición. Alianza Editorial. Madrid, España.

CEPAL. 2010. "Agenda 2030 para el Desarrollo Sostenible" En: CEPAL Biblioguías. Disponible en línea. En: https://biblioguias.cepal.org/ agenda2030. Fecha de consulta: 12/19/2018.

CEPAL. 2018. "Construcción del Índice de Gobierno Abierto" In: Informe de Luis Paternia de la Red de Planificación para el desarrollo en América Latina y el Caribe. Disponible en línea. En: https://www.cepal.org/sites/ default/files/igao2_antecedentes.pdf. Fecha de consulta: 31/o9/2019.

CRIADO, J. Ignacio; RUVALCABA-GÓMEZ, Edgar Alejandro. 2016. ¿Qué es y qué se entiende por gobierno Abierto? Análisis de la percepción e implementación del Gobierno Abierto en el ámbito local español. Laboratorio iberoamericano de gobierno para la innovación pública. Madrid, España.

CRIADO, J. Ignacio; RUVALCABA-GÓMEZ, Edgar Alejandro; VALENZUELAMENDOZA, Rafael. 2018. "Revisiting the Open Government Phenomenon. A Meta-Analysis of the International Literature" En: JeDEM - EJournal of EDemocracy and Open Government. Vol. 10, No. 1 , pp. 50-81. 
Lorayne Finol Romero
46 Transparencia y Gobernanza en la Gestión de la Crisis de COVID-19

DIAMOND, Larry. 2003. "¿Puede el mundo entero ser democrático? Democracia, desarrollo y factores internacionales” En: Revista Española de Ciencia Política. No. 9, pp.9-38.

FINOL, Loraine. 2018. "Una aproximación teórica al gobierno abierto" En: Cuestiones Políticas. Vol. 34, No. 60, pp. 69-91.

FINOL, Loraine. 2020. “Transparentando la transparencia en tiempo de COVID 19" En: Ponencia presentada en Seminario: Pandemia, Transparencia y datos abiertos. Universidad Central de Chile. Santiago de Chile, Chile.

FLORES-CRESPO, Pedro. 2013. "El Enfoque de la Política Basado en la Evidencia: Análisis de su utilidad para la educación de México" En: Revista Mexicana de Investigación educativa. Vol. 18, No. 56, pp. 265290.

FORERO DE MORENO, Isabel. 2009. "La Sociedad Del Conocimiento" En: Revista Científica General José María Córdova. Vol. 5, No. 7, pp. 40-44.

GARGARELLA, Roberto. 2015. "El nuevo constitucionalismo Latinoamericano" En: Estudios Sociales. Revista Universitaria Semestral. Vol. 48, No. 1, pp. 169-174.

GUICHOT REINA, Emilio. 2012. "La transparencia en España: estado de la cuestión” En: Tabula: Revista de archivos de Castilla y León. No.15, pp. 259-290.

HOLLYER, James R; Rosendorff, Peter, B; VREELAND, James R. 2011. "Democracy and Transparency" En: The journal of politics. Disponible en línea. En: https://doi.org/10.1017/Soo22381611000880. Fecha de consulta: 08/04/ 2019.

LATHROP, Daniel; RUMA, Laura. 2010. "Open government: Collaboration, transparency, and participation in practice”. O’Reilly Media, Inc. Nueva York, EE. UU.

MARGETTS, Helen; DOROBANTU, Cosmina. 2019. "Rethinking government with AI” En: Nature. Disponible en línea. En: Doi: 10.1038/d41586-01901099-5. Fecha de consulta: 11/04/2019.

MATHEUS, R; JANSSEN, M. 2019. "Un estudio sistemático de literatura para desentrañar la transparencia habilitado por los datos de gobierno abierto: The Window Theory” En: Public Performance \& Management Review. No. 42, pp. 1-33.

MEIJER, Albert; HART, Paul 't; WORTHY, Ben. 2018. “Assessing Government Transparency: An Interpretive Framework" En: Administration \& Society. Vol. 50, No. 4, pp. 501-526. 
MINISTERIO DE LA SECRETARÍA DE LA PRESIDENCIA DE CHILE. 2018. Cuarto Plan Nacional de Acción corresponde al cuarto comprometido por Chile, abarcando el período 2018-2020. Comisión de Integridad Pública y Transparencia. Disponible en línea. En: https://www. opengovpartnership.org/wp-content/uploads/2019/01/Chile_ActionPlan_2018-2020.pdf. Fecha de consulta: 25/06/2018.

MOORE, Mark H. 1997. Creating Public Value Strategic Management in Government. Harvard University Press. Cambridge, Estados Unidos.

MORLINO, Leonardo. 2007. "Explicar la calidad democrática: ¿qué tan relevantes son las tradiciones autoritarias?” En: Revista de Ciencia Política. Vol. 27, No. 2, pp. 3-22.

MUELLER, Christoph; ENGEWALD, Bettina. 2018. "Making Transparency Work: Experiences from the Evaluation of the Hamburg Transparency Law” En: Central European Public Administration Review. Vol. 16, No. 2, pp. 69-90.

MUENTE, Arturo; SERALE, Florencia. 2019. "Los datos abiertos en América Latina y el Caribe" Banco Interamericano de Desarrollo. Disponible en línea. En: http://dx.doi.org/10.18235/0001202. Fecha de consulta: 12/03/2019.

MULGAN, Geoff. 2016. "Los desafios para innovar en el sector público. Exigencias, beneficios y aproximaciones para innovar creando valor público" En: Ponencia presentada en la conferencia Internacional de Innovación Pública. Santiago de Chile, Chile.

NASER, Alejandra; CONCHA, Gastón. 2012. "Datos abiertos: Un nuevo desafío para los gobiernos de la región" En: Instituto Latinoamericano y del Caribe de Planificación Económico y Social (ILPES) de las Naciones Unidas. Santiago de Chile. Disponible en línea. En: https://repositorio. cepal.org/bitstream/handle/11362/7331/1/S1200084_es.pdf. Fecha: 09/02/2019.

NASER, Alejandra; ROSALES, Daniela. 2016. "Panorama regional de los datos abiertos Avances y desafíos en América Latina y el Caribe" En: Publicación de la Comisión Económica para América Latina (CEPAL) de las Naciones Unidas. Disponible en línea. En: https://www.cepal.org/ es/publicaciones/40768-panorama-regional-datos-abiertos-avancesdesafios-america-latina-caribe. Fecha de consulta: 05/12/2017.

OBAMA, Barack. 2009. "Memorandum Transparency and Open Government". En: The White House. Disponible en línea: https:// obamawhitehouse.archives.gov/the-press-office/transparency-and- 
Lorayne Finol Romero
48 Transparencia y Gobernanza en la Gestión de la Crisis de COVID-19

open-government\#: :text=Openness\%20will\%2ostrengthen\%20 our\%20democracy,Government\%20is\%20a\%2onational\%20asset. Fecha: 09/02/2019.

OCDE. 2020. "OECD Open, Useful and Re-usable data (OURdata) Index: 2019" En: OECD Publishing, Paris. Disponible en línea. En: http://www.oecd. org/gov/digital-government/. Fecha de consulta: 15/10/ 2020.

OPEN GOVERNMENT DATA ACT. 2019. "Ley de fundamentos para la formulación de políticas basada en evidencia del 2018”. Núm. 115-11. Fecha 15 de febrero de 2019. Promulgación HR 4174.

OPEN GOVERNMENT PARTNERSHIP [OGP]. 2011. Alianza para el gobierno abierto. Declaración de gobierno Abierto. Disponible en línea. En: https://www.opengovpartnership.org/. Fecha de consulta: 20/11/2017.

OPENDATA CHARTER. 2015. Carta internacional de los Datos Abiertos. Disponible en línea. En: https://opendatacharter.net/principles-es/. Fecha de consulta: 15/10/2018.

ORGANIZACIÓN DE LAS NACIONES UNIDAS. 2015. Objetivos para el Desarrollo Sostenible [ODS]. Agenda para el desarrollo sostenible 2030. Disponible en línea. En: https://www.un.org/sustainabledevelopment/. Disponible en línea. En: 20/11/ 2019.

PARLAMENTO EUROPEO. 2003. Directiva 2003/98/CE Del Parlamento Europeo y del Consejo de 17 de noviembre de 2003 relativa a la reutilización de la información del sector público. Publicada en el Diario Oficial de la Unión Europea. Fecha 31/12/2003. Disponible en línea. En: https://www.boe.es/doue/2003/345/Lo0090-00096.pdf. Fecha de consulta: 08/04/ 2017.

PARLAMENTO EUROPEO. 2019. Directiva (UE) 2019/1024 del Parlamento Europeo y del Consejo, de 20 de junio de 2019, relativa a la reutilización de la información del sector público. Publicada en el Diario Oficial de la Unión Europea. Bruselas, Bélgica.

QUINTANILLA, Gabriela; GIL-GARCÍA, José. 2016. “Gobierno Abierto y datos abiertos vinculados: conceptos, experiencias y lecciones con base al caso mexicano" En: Revista del CLAD Reforma y Democracia. No. 65, pp. 69102.

RODRÍGUEZ-ZEPEDA, Jesús. 2008. "Estado y transparencia: un paseo por la filosofía política” En: Cuaderno de Transparencia 4. Instituto Federal de Acceso a la Información Pública. México DF., Mexico. 
ROELOFS, Portia. 2019. "Transparency and mistrust: Who or what should be made transparent?” En: Governance. Disponible en línea. En: https:// doi.org/10.1111/gove.12402. Fecha de consulta: 02/03/2020.

RUIJER, Erna; MEIJER, Albert. 2016. "Regímenes nacionales detransparencia: ¿Reglas o principios? Un análisis comparativo de los Estados Unidos y los Países Bajos" En: International Journal of Public Administration. Vol. 39, No. 11, pp. 895-908.

RUVALCABA GÓMEZ, Edgar A. 2020. "Datos abiertos open data” En: Eunomía Revista en Cultura de la Legalidad. Disponible en línea. En: doi: https:// doi.org/10.20318/eunomia.2020.5280. Fecha de consulta: 12/19/2020.

SAFAROV, Igbal (2019), "Institutional Dimensions of Open Government Data Implementation: Evidence from the Netherlands, Sweden, and the UK" En: Public Performance \& Management Review. Vol. 2, No. 2, pp. 305328.

SAFAROV, Igbal; MEIJER, Albert; GRIMMELIKHUIJSEN, Stephan. 2017. "Utilization of open government data: A systematic literature review of types, conditions, effects, and users" En: Information Polity. Vol. 22, No. 1, pp. 1-24.

SANDOVAL-ALMAZÁN, Rodrigo; STYRIN, Evgeny. 2018. "Towards an Open Government Data Comparative Model" En: Proceedings of the 11th InternationalConferenceonTheoryandPracticeofElectronicGovernance. Disponible en línea. En: https://doi.org/10.1145/3209415.3209434. Fecha de consulta:15/09/2019.

SANTA, Ricardo; MACDONALD, Jason; FERRER, Mario. 2019. "The role of trust in e-Government effectiveness, operational effectiveness and user satisfaction: Lessons from Saudi Arabia in e-G2B” En: Government Information Quarterly. Disponible en línea. En: https://doi. org/10.1016/j.giq.2018.10.007. Fecha de consulta: 13/02/2020.

SCROLLINI, Fabrizio. 2017. Evaluación de la transparencia: estado del arte de indicadores. Consejo para la Transparencia. Santiago de Chile, Chile.

SHAMBAUGH, George; SHEN, Elaine. 2018. "A clear advantage: The benefits of transparency to crisis recovery". En: European Journal of Political Economy. No. 55, pp. 391-416.

STRAUSS, Anselm; CORBIN, Juliet. 2002. Bases de la investigación cualitativa. Técnicas y procedimientos para desarrollar teoría fundamentada. Universidad de Antioquia. Medellín, Colombia. 
TAPSCOTT, Don; AGNEW, David. 1999. "Governance in the Digital Economy" En: Finance and Development. Vol. 36, No. 4, pp. 34-37.

TORRES, Natalia. 2014. "Hacia una política integral de información pública" El acceso a los archivos: sistemas de gestión de la información implementados en América Latina. Universidad de Palermo, pp. 11-46.

WORLD WIDE WEB FOUNDATION. 2018. "Open Data Barometer Global Report (Cuarta edición)”. Disponible en línea. En: http://www. opendatabarometer.org. Fecha de consulta: 12/03/2019.

WORTHY, Benjamin. 2015. "El impacto de los datos abiertos en el Reino Unido: complejo, imprimible y político” En: Public Administration. Vol. 93, No. 3, pp. 788-805. 

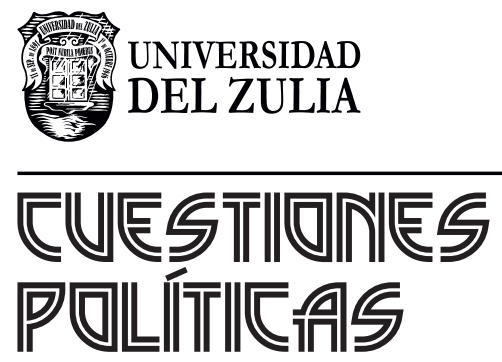

Vol.39 No 68

Esta revista fue editada en formato digital y publicada en enero de 2021, por el Fondo Editorial Serbiluz, Universidad del Zulia. Maracaibo-Venezuela 